Revista de Filosofía

ISSN: 0034-8244

http://dx.doi.org/10.5209/RESF.64274

\title{
Materialismo e artefattualità. Una filosofia politica della materia
}

\author{
Ernesto Sferrazza Papa ${ }^{1}$
}

Recibido: 30 de agosto de 2017 / Aceptado: 09 de octubre de 2018

Riassunto. In questo saggio analizzo l'idea del mondo come coesistenza di umani e cose, e sostengo che tale coesistenza debba essere considerate sotto la lente concettuale della responsabilità. Nei primi due paragrafi sostengo che l'esperienza umana è sempre mediata da un sistema di artefatti. Nel terzo e nel quarto paragrafo sintetizzo due differenti approcci filosofici, ossia la teoria degli artefatti e il metodo materialista, $\mathrm{i}$ quali condividono la tesi della performatività degli artefatti nella costituzione dell'esperienza umana. Nelle conclusioni mostro la rilevanza etica e politica di questa possibile ermeneutica materiale del mondo.

Parole chiave: umano; artefatto; materialismo; ermeneutica; responsabilità.

\section{[en] Materialism and Artefactuality. A Political Philosophy of the Topic}

\begin{abstract}
In this paper, I analyze the idea of the world as the co-existence of humans and things. I argue that this co-existence should be considered from the conceptual perspective of responsibility. In the first two paragraphs, I argue that a system of artefacts always mediates human experience. In the third and fourth paragraphs, I synthetize two different philosophical approaches, namely the theory of artefacts and the materialistic method, which share the thesis of the performativity of artefacts in the constitution of human experience. In the conclusions, I show the political and ethical relevance of this possible material hermeneutics of the world.
\end{abstract}

Keywords: human; artefact; materialism; hermeneutics; responsibility.

Sumario: 1. Le partage du sensible; 2. L'oggetto come mediator; 3. Una politica della materia: Simone Weil e Günther Anders; 4. La politicità dell'artefatto; 5. Conclusioni: una filosofia politica e morale della materialità; 6 . Riferimenti bibliografici.

Cómo citar: Sferrazza Papa, E. (2019): "Materialismo e artefattualità. Una filosofia politica della materia", en Revista de Filosofia 44 (1), 113-129.

1 Instituto de Filosofía, Facultad de Filosofía

Pontificia Universidad Católica de Chile (Chile)

ernesto.sferrazzapapa@unito.it 


\section{Le partage du sensible}

Lo spazio del vivente, il suo Lebensraum, è un mondo costituito essenzialmente da oggetti ${ }^{2}$. Se la realtà, come è stato sostenuto, è quell'esistente che oppone una resistenza nei confronti dell'azione dei viventi e dei loro schemi concettuali (Ferraris 2012), ciò avviene in primo luogo perché è composta da oggetti inanimati, inorganici, da ciò che apparentemente tutto separa dal vivente. I viventi sono prima di tutto "ospiti di un mondo di cose" (Leghissa 2014, p. 10). Esse rappresentano il limite ultimo dell'azione del soggetto. La realtà materiale si presenta così allo stesso tempo come condizione materiale di possibilità dell'esistenza del soggetto e limite invalicabile della sua esperienza. L'esperienza primaria del vivente è da rintracciarsi prima di tutto nel contatto con la materia, con gli oggetti: come scrive Ian Hodder, "ogni essere senziente dipende dalle cose per portare la sua senzienza (sentience) all'essere" (Hodder 2012, p. 9). L'individuo diviene realmente tale nel momento in cui fa esperienza della consistenza del mondo che lo circonda; lo tocca, vi sbatte contro, ne constata la resilienza, ne comprova la resistenza. L'esperienza fondamentale del vivente è la percezione di qualcosa che provoca attrito. Montagne, laghi, fiumi, muri, case, tavoli: ovunque si volga lo sguardo del vivente, sempre incontra oggetti, res inanimatae a sbarrargli, prima o poi, la strada, o a costringerlo a modificare la traiettoria del suo cammino, a indirizzare e organizzare il suo agire.

È necessaria una preliminare chiarificazione concettuale e linguistica per distinguere tre termini che nel linguaggio ordinario tendenzialmente vengono confusi: cosa, oggetto e artefatto. Essi comportano sfumature di senso che obbligano a distinguere recisamente quantomeno il primo dagli altri due. Remo Bodei rileva che "cosa è, per certi versi, l'equivalente concettuale del greco pragma, della latina res o del tedesco Sache [...], parole che non hanno niente a che vedere con l'oggetto fisico in quanto tale e neppure con l'uso corrente del tedesco Ding o dell'inglese thing [...], ma che contengono tutte un nesso ineliminabile non solo con le persone, ma anche con la dimensione collettiva del dibattere e del deliberare" (Bodei 2009, pp. 12-13). Una cosa è ciò che viene investito e caricato di un interesse da parte di un soggetto. Essa implica tutto un fascio di relazioni, di affetti, che la legano al soggetto. Le cose sono dunque ciò su cui si orienta il nostro interesse, singolare o collettivo. In questo senso si parla ad esempio di respublica, la cosa pubblica, ossia di ciò su cui si cristallizza l'interesse di una collettività.

La nozione di oggetto ha una storia filosofica differente che affonda radici nella scolastica medievale. Un oggetto è innanzitutto un ostacolo, un problema, qualcosa

\footnotetext{
Se volessimo tracciare una grammatica filosofica dell'oggettualità, dovremmo risalire alla replica che Parmenide, nel dialogo platonico omonimo, muove a Socrate quando quest'ultimo rifiuta di concepire l'esistenza di forme separate rispetto a "oggetti che sembrerebbero ridicoli come capello, fango, sporco, oppure qualcos'altro senza valore e di pochissimo conto" (Prm. 130c). Agli oggetti, questo il senso, va in ogni caso riconosciuta una dignità ontologica. Stando così le cose, la postura assunta dalla riflessione filosofica contemporanea sugli oggetti, come vedremo nella parte finale del saggio, è coerentemente decentrata rispetto alla prospettiva antropocentrica. Michel Foucault ad esempio definisce l'uomo - colui che nomina le cose - "una certa lacerazione nell'ordine delle cose" (Foucault 2010, p. 13): le cose rappresentano una zona mediana, pre-linguistica, nella quale il vivente si dà come ente fra gli enti. L'uomo è una breccia di quest'ordine, una sua declinazione piuttosto che il suo latore di senso. La corda tesa che va da Platone ai vari Heidegger, Foucault, Simondon, Baudrillard, Weil, Benjamin, Deleuze, Latour, le cui opere rappresentano le tappe di questa genealogia del pensiero sull'inerte, può dunque essere ulteriormente tirata in direzione di una metafisica non antropocentrica che, questa la mia tesi, riconosce almeno una prerogativa all'umano, ossia la proprietà di essere un agente morale.
} 
contro cui l'esperienza del soggetto va a sbattere. L'oggetto è dunque ciò che si contrappone al soggetto; esso è "l'ostacolo indeterminato che ho di fronte e che devo abbattere o aggirare" (Bodei 2009, p. 20). Possiamo considerare l'oggetto come un aggregato di materia che si contrappone in quanto tale alla libertà del soggetto, al suo agire. Esso esprime la resistenza del reale nei confronti dell'attività umana e del vivente tout court. Non è primariamente qualcosa su cui facciamo convergere il nostro interesse (per quanto ciò possa avvenire tramite un investimento di interesse che trasforma l'oggetto in cosa), ma è qualcosa che anzitutto si frappone alla nostra esperienza, la ostacola ma, come vedremo in seguito, allo stesso tempo la organizza e la rende possibile.

Qual è lo statuto ontologico dell'artefatto? La Stanford Encyclopedia of Philosophy propone una definizione di artefatto che, nonostante qualche doverosa cautela relativa al riferimento per nulla ovvio alla nozione di intenzionalità, può essere in via preliminare accettata: "un artefatto è un oggetto che è stato intenzionalmente fatto o prodotto per un certo scopo". L'artefatto è dunque una sottocategoria dell'insieme degli oggetti, e potremmo definirlo come un oggetto fabbricato, il risultato materiale dell'attività dell' Homo faber.

L'essere umano è dunque parte di un sistema di oggetti che ha egli stesso in larga parte prodotto. Prima di esservi un sistema di cose, vi è un sistema di oggetti. La Lebenswelt del vivente è il regno della datità materiale con la quale egli si trova in ogni momento ad avere a che fare, a esserle costantemente entangled (Hodder 2012). Benché sia in linea di principio perfettamente possibile separare e isolare la componente biologica, dunque puramente naturale del corpo del vivente, pensandolo come un determinato aggregato di cellule destinato inesorabilmente a disfarsi, è problematico immaginare un'esistenza - quantomeno umana - che sin dal primo respiro non sia in comunicazione con il mondo dell'oggettuale, con ciò che resisterà anche dopo la fine dell'uomo.

Prima di essere il teatro dove è messa in scena la commedia umana, il mondo è dunque un palcoscenico abitato da oggetti con i quali gli esseri umani devono in ogni momento fare i conti. All'interno di questa sterminata molteplicità di oggetti che determinano il Dasein del vivente uomo, numerose linee di separazione possono essere tracciate. Sin dalle origini del pensiero occidentale, la filosofia si è costituita come una riflessione sul mondo, ossia su ciò che esiste e su ciò che non esiste, e da questa prospettiva privilegiata si è interrogata sulle caratteristiche degli elementi che costituiscono il nocciolo ultimo di ciò che chiamiamo realtà. La conoscenza delle cause delle cose è da sempre stata presa in carico dalla filosofia, e qualunque incursione nel pensiero classico può testimoniarlo. Quando le dottrine metafisiche dei presocratici hanno assegnato ad elementi (acqua, terra, fuoco, pneuma) il ruolo di archè, di origine, ossia di elementi costitutivi ultimi della realtà, non hanno fatto altro che domandarsi quali fossero le cause e le modalità di articolazione di quello che Jacques Rancière chiamerebbe le partage du sensible (Rancière 2000). Qui si può situare un problema teorico di primaria importanza, che ha trovato nella riflessione ontologica in generale e nella sua rinascita contemporanea in particolare un tentativo di soluzione, o quantomeno di risposta. Sostenere che il sensibile, ossia che lo spettro di ciò che si dà all'esperienza, sia ripartito, ossia articolato in un certo modo, implica la necessità filosofica di rendere conto dei motivi di tale ripartizione. Infatti, che una cosa sia separata da un'altra non è affatto un'ovvietà, ma sottende una visione metafisica che giustifica la loro determinazione reciproca. 
Per approcciare il problema mi servirò, in maniera senza dubbio strumentale, di un passaggio della Fisica aristotelica. Aristotele ha imbastito nel corso della sua riflessione filosofica un catalogo del mondo, ossia un tentativo di spiegare le cause di questa apparentemente ovvia partizione del sensibile, tracciando una separazione fondamentale tra oggetti naturali e artefatti. Questa divisione è illustrata all'inizio del II libro della Fisica, dove Aristotele pone una differenza fra oggetti esistenti per natura e oggetti esistenti per altre cause, ossia fra oggetti naturali e oggetti tecnici: "[d] egli enti, alcuni sono per natura, altri sono dovuti ad altre cause" (Phys., $192 \mathrm{~b}$ 10). La distinzione tracciata da Aristotele è metafisica: concerne le cause per cui un ente è tale. Mentre un oggetto per natura ha "in se stesso il principio del movimento e della quiete" (Phys., 192 b 10), un oggetto della seconda classe non possiede "alcuna

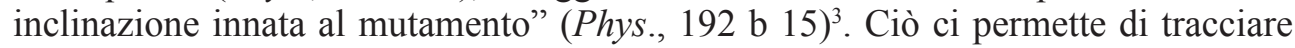
una prima e grossolana distinzione, ripartendo il mondo tra ciò che è stato costruito e ciò che non lo è stato. Questa abbozzata ontologia è per molti versi problematica ${ }^{4}$, ma ha il pregio di chiarire con che tipo di oggetti abbiamo a che fare quando ci muoviamo nel mondo. Se è vero che abitiamo sin dal principio un mondo di oggetti naturali, il nostro rapporto con esso è pur sempre costantemente mediato da artefatti, ossia da oggetti prodotti dallo sforzo umano di manipolare tecnicamente una base materiale. Dipendiamo dagli oggetti, ma gli oggetti dipendono da noi.

A partire da queste considerazioni, volte più che altro a tracciare una cornice ermeneutica nella quale situare una serie di problemi, lo sforzo del presente contributo sarà quello di indagare le possibili implicazioni, da un punto di vista sia ontologico sia politico, del riconoscimento del mondo come coesistenza di viventi e artefatti ${ }^{5}$. Ritengo che tale prospettiva possa avere un risvolto particolarmente interessante dal punto di vista della ricerca filosofica, giacché a partire da questa constatazione del

3 Nonostante il richiamo a questo passaggio aristotelico sia per me meramente strumentale, è in ogni caso necessario un chiarimento intorno ai concetti di movimento (kínesis) e mutamento (metabolé) in Aristotele. In alcuni luoghi del corpus aristotelico, come ad esempio nel passo citato della Fisica e in alcuni luoghi della Politica (II, 8, 1268 b 25; IV, 16, 1300 b 38; V, 2, 1302 a 34-35), Aristotele non distingue i due termini, che possono essere interpretati dunque come sinonimi. Tuttavia, in un topos cruciale per il problema del movimento nella teoria aristotelica quale Categorie 14, lo Stagirita distingue esplicitamente il movimento dal mutamento secondo il luogo (kata topon metabole: 15a 13-14). Il mutamento secondo il luogo diventa qui una delle sei specie del movimento (generazione, corruzione, aumento, diminuzione, alterazione). Ciò fa pensare che i due termini non possano essere sovrapposti. I commentatori, a partire da Simplicio fino ad arrivare alle esegesi contemporanee, hanno offerto interpretazioni differenti di questo oscillamento semantico, e non è questa la sede per ripercorrerle. Per una discussione lucida e aggiornata del problema si veda Salis (2011).

4 Ovviamente, l'ontologia aristotelica è solo un primo e accennato tentativo di descrivere questa partizione del sensibile. Non è possibile ricondurre sempre gli oggetti che popolano il mondo, e con i quali il vivente costantemente si rapporta, a questa duplicità. L'ontologia sociale, in particolare, ha preso in carico l'analisi e la definizione di tutta una serie di oggetti quali le promesse, i debiti, i matrimoni, le lezioni universitarie, le monete, i valori di scambio, che organizzano quotidianamente le nostre esistenza, ma che non si lasciano ricondurre alla bipartizione aristotelica. Gli oggetti che definiscono il nostro mondo sociale necessitano di una prospettiva ontologica più raffinata, sulla quale tuttavia non ci soffermeremo in questa sede. Per una panoramica dei problemi affrontati e sollevati dall'ontologia sociale si veda Ferraris 2008.

5 Nonostante alcuni punti comuni, la prospettiva teorica che intendo adottare non è riconducibile all'Action Network Theory (ANT), il cui Diòscuro è il sociologo e filosofo francese Bruno Latour (2005). L'ANT infatti si basa sul principio della simmetria generalizzata. Secondo tale principio, la capacità di agency degli umani e degli artefatti deve potere essere analizzata in maniera perfettamente simmetrica. Contrariamente ai sostenitori dell'ANT, io sostengo che l'azione umana mantenga una specificità inaggirabile rispetto a quella degli artefatti, che risiede nella capacità di interrogarsi sull'azione stessa, sul valutare dunque non solamente il rapporto tra mezzo e fine, ma il significato di entrambi i termini. In questo modo ritengo sia possibile garantire all'umano una specificità, ossia quella di essere un agente morale, senza compromettere la tesi dell'agency degli artefatti. 
mondo come aggregato di molteplici forme di vita, animate e inanimate, operose e inerti, attive e passive, svilupperò la tesi di una possibile sintesi fra la riflessione ontologica sugli artefatti e alcune teorie filosofiche che hanno fatto proprio il metodo materialista.

\section{L'oggetto come mediator}

Gli artefatti costituiscono il medium della nostra esperienza con il mondo. Essi sono il punto in cui le potenzialità del nostro corpo, in primo luogo muoversi e manipolare la materia, entrano in comunicazione con l'ambiente nel quale vengono effettivamente realizzate. Senza dubbio alcuno, condividono questa proprietà mediale con il regno degli oggetti naturali; tuttavia, a differenza di un oggetto naturale, un artefatto viene deliberatamente prodotto al fine di essere il tramite, l'anello di congiunzione, tra la volontà del vivente - che tenta di esteriorizzare, ossia di realizzare, un proprio fine specifico - e la datità del mondo nella quale tale volontà deve sperabilmente essere realizzata. Il gesto per eccellenza dell'umano si configura nella forma di una manipolazione cosciente e intenzionale del mondo esterno, di un uso delle mani volto alla produzione di un universo di artefatti convocati a mediare, ossia a rendere possibile in un modo determinato, una certa forma di rapporto con il mondo.

Approfondiamo la nozione di medium. Medium è letteralmente ciò-che-sta-inmezzo, è l'elemento terzo che si frappone fra i due termini di una relazione. Medium è, dunque, il punto medio di tale rapporto. Allo stesso tempo però, il medium è anche un dispositivo (da dispositio, il disporre le cose secondo un determinato ordine) che istituisce le modalità attraverso le quali di tale rapporto è possibile fare esperienza. Se sta in mezzo a due termini, ossia se li media, è perché è in grado di determinarne la disposizione.

La nozione di medio fa capolino per la prima volta nella storia della filosofia occidentale con la logica aristotelica. Nei Primi Analitici, trattando degli elementi che compongono un sillogismo, Aristotele definisce medio quel termine che "tanto è contenuto esso stesso in un altro termine, quanto contiene in sé un altro termine, e che si presenta come medio anche per la posizione" (An. pr., 25 b 35). Il medio dunque è ciò che, mettendo in comunicazione due estremi, permette il sillogismo, ossia la loro relazione logica.

Se trasportiamo l'argomento aristotelico al rapporto tra uomini e oggetti, il medium deve essere considerato come un dispositivo che, articolando l'esperienza che l'essere umano fa del mondo, la rende possibile in una forma specifica, ossia ne definisce allo stesso tempo i limiti materiali. Un esempio molto semplice chiarirà questo punto: gli occhiali sono ciò che, frapponendosi tra l'occhio e il campo visivo, rende possibile l'esperienza visiva; allo stesso tempo, la conformazione materiale degli occhiali rende possibile solo una determinata esperienza visiva e non altre (ad esempio, degli occhiali da vista non permetteranno di guardare direttamente il sole, mentre gli occhiali da sole renderanno possibile per qualche secondo questa esperienza).

Se sono gli oggetti in quanto media a rendere possibile il rapporto dell'essere umano con il mondo, allora il monito husserliano sulla necessità di ritornare alle "cose stesse" assume in questa sede una veste tutta particolare. Dalla nostra specola, invece che alle cose stesse, è opportuno ritornare agli oggetti stessi, ossia 
a riconoscere una dignità ontologica, fenomenologica e metafisica non solo al vivente in generale, ma alla datità puramente materiale, all'inorganico, ossia a quel medium che organizza l'esperienza del vivente con il mondo esterno. Trasportando in questo contesto la terminologia biblica (sperando che l'operazione non risulti eccessivamente pretenziosa), l'oggetto materiale deve essere inteso come mediator mundi et hominum ${ }^{6}$. Ogni oggetto infatti può essere considerato un medium nel momento in cui articola un certo modo del vivente di fare esperienza del mondo. In questo senso, Walter Benjamin parla del medium come del "modo secondo cui si organizza la percezione sensoriale umana" (Benjamin 2012b, p. 21). Un colore, "puro accidente privo di sostanza" (Benjamin 2012a, p. 92), può essere un medium, così come un dispositivo tecnico - ad esempio l'apparecchiatura fotografica. In ogni caso, medium è sempre ciò che organizza e rende possibile l'esperienza che il vivente fa del mondo, un'esperienza che viene così a essere sia naturale - nel

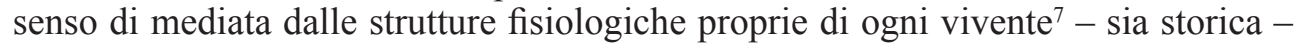
nel senso di mediata dallo sviluppo tecnologico. L'esperienza estetica, considerata nella sua accezione baumgartiana di scienza della conoscenza sensibile, quella che in un saggio recente Emanuele Coccia ha chiamato la vita sensibile (Coccia 2011), ha come condizione di possibilità l'esistenza di un sistema di oggetti che articola il rapporto del vivente con il mondo.

\section{Una politica della materia: Simone Weil e Günther Anders}

Due campi della ricerca filosofica sembrano convergere su questo campo della datità materiale, senza tuttavia essersi mai realmente incontrati. Da un lato, la teoria degli artefatti, che riconosce agli artefatti uno statuto ontologico autonomo in virtù delle proprietà materiali che li separano, ad esempio, dagli oggetti sociali e da quelli ideali; dall'altro lato, il metodo materialista, che considera la struttura materiale del mondo, e dunque le condizioni oggettive dei modi di produzione, primaria rispetto alle condizioni sociali e culturali, ossia rispetto alla sovrastruttura. La mia tesi è che queste due posture filosofiche condividano, oltre all'oggetto di indagine - il dato materiale, ciò che è per eccellenza sensibile -, anche una reciproca mancanza che solo attraverso la loro sintesi può essere colmata. Infatti, mentre la teoria degli artefatti tende a non considerare le implicazioni politiche e sociali degli artefatti che analizza, tentando piuttosto di sviluppare una visione teorica generale e avendo quasi sempre come riferimenti esemplari oggetti semplici le cui proprietà causali sono immediatamente individuabili (caffettiere, sedie, martelli), l'approccio materialista, che insiste viceversa sulla politicità delle condizioni materiali che articolano la realtà, difetta di una vera e propria teoria dell'oggetto, dando per scontata quella

6 Stando così le cose, la celebre affermazione di McLuhan, the medium is the message - sulla quale McLuhan fu costretto più volte a ritornare per dipanare i numerosi fraintendimenti che andavano accumulandosi su essa - va compresa nella sua accezione prettamente pragmatista di ascendenza peirciana. Dire che il medium è il messaggio significa essenzialmente che il medium (e la proprietà di medium può essere estesa secondo McLuhan a tutto il reale) produce determinate conseguenze nelle modalità di esistenza di chi esperisce il rapporto con il mondo per suo tramite: "il messaggio di un medium o di una tecnologia è nel mutamento di proporzioni, di ritmo o di schemi che introduce nei rapporti umani” (McLuhan 1990, p. 15).

7 In questo senso va concepito il "kantismo fisiologico" che sta alle base delle riflessioni sul rapporto fra organismo e ambiente (Umwelt) di Uexküll (1934). 
partizione del sensibile che costituisce la base di ogni possibile ontologia. Si tratta dunque di chiarire per quale motivo queste direzioni della ricerca filosofica, che una distinzione che ritengo anacronistica tra filosofia analitica e continentale inviterebbe a pensare come separate, convergano in realtà su un problema teorico comune - la natura dell'artefatto e il suo ruolo nell'esperienza del vivente -, in modo da potersi reciprocamente completare.

A questo scopo, vorrei servirmi di alcune intuizioni del marxismo eterodosso di Simone Weil. Ella porta agli estremi l'approccio materialista di Marx, piegandolo direttamente sull'organizzazione artefattuale del mondo. L'elemento strutturale diviene in Weil la struttura concreta, artefattuale, dell'ambiente abitato dall'umano. A tal proposito, è istruttiva una boutade di Étienne Balibar secondo cui "il materialismo di Marx non ha niente a che fare con un riferimento alla materia [...]. Abbiamo a che fare con uno strano «materialismo senza materia»" (Balibar 1993, p. 24). Si potrebbe replicare a Balibar che il suo è un trucco di prestigio che fa giocare i termini "materia" e "materialismo", ma vorrei essere più tranchant e mostrare che la sua affermazione è semplicemente falsa. Infatti, già nel Frammento sulle macchine Marx aveva riconosciuto agli artefatti determinate proprietà implicite nella loro materialità; il suo materialismo dunque, pur non essendo il materialismo intuitivo degli antichi - che lo stesso Marx critica a più riprese -, attinge a piene mani dal mondo della datità materiale. Non è vero insomma che quello di Marx sia un materialismo senza materia (cfr. MacKenzie 1984). Nel Frammento sulle macchine Marx afferma che la gamma di azioni possibili della macchina rimane estranea alla volontà dell'operaio perché ha a che fare con "il potere della macchina stessa" (Marx 1970, p. 391), presupponendo così una teoria della macchina come artefatto dotato di proprietà causali intrinseche. La macchina ha in quanto macchina un potere che organizza le possibilità dell'operaio. La sua struttura fisica determina specifiche costrizioni dell'attività umana: se il potere della macchina non deriva dall'operaio che l'aziona, allora logicamente deriva da alcune proprietà che sono implicite nella sua materialità. Ciò suggerisce la necessità di riflettere su quali siano le proprietà di un artefatto in quanto tale, considerandolo cioè nel suo valore puramente strutturale. Già Marx insomma aveva esplicitamente indicato che vi è un nesso fra il mezzo e il fine, che il risultato del lavoro non è autonomo bensì eteronomo rispetto alla macchina che lo rende possibile. Questa intuizione viene esplicitamente ripresa dall'ermeneutica materialista eterodossa di Simone Weil.

Simone Weil compendia il suo metodo materialista, che considera primarie per l'analisi di un fenomeno le condizioni di possibilità nelle quali esso si presenta, in un passaggio delle Réflexions sur la guerre: "esaminare qualunque fatto umano tenendo conto assai più delle conseguenze necessariamente implicite nel gioco dei mezzi adottati che dei fini perseguiti" (Weil 2005, p. 31). Secondo Weil il metodo materialista ha come campo d'analisi in primo luogo la datità materiale per come essa si presenta all'esperienza umana. Esso, in generale, indaga quali siano le relazioni fra le determinazioni materiali che compongono una società e i rapporti sociali che la costituiscono e l'attraversano. Il monito metodologico di Weil coglie perfettamente la possibilità di una prospettiva sintetica, ancora inesplorata, che permetta di saldare il metodo materialista con la teoria ontologica degli artefatti. La necessità di tracciare le coordinate teoriche di questo legame emerge con maggior forza in un altro testo di Weil, le Réflexions sur les causes de la liberté et de l'oppression sociale, dove viene chiarito il bisogno politico di un'ermeneutica materiale. Quando Weil, criticando 
il marxismo, colpevole a suo dire di non aver indagato fino in fondo le cause dell'oppressione sociale, afferma la necessità di un'analisi delle condizioni materiali che garantiscono o meno la possibilità di un'azione emancipatrice nei confronti della realtà oppressiva del proletariato, sfiora quella teoria ontologica degli artefatti che l'approccio materialista dovrebbe radicalmente fare propria.

La critica di Weil al marxismo non ne nega i presupposti, bensì li radicalizza. A suo parere, il marxismo non avrebbe scavato fino in fondo nell'assunto del carattere necessitante dei mezzi materiali. Anche secondo Weil infatti le cause dell'oppressione sociale sono da ricercarsi in primo luogo nelle condizioni materiali di sfruttamento, dove l'aggettivo "materiale" deve essere immediatamente riferito alle implicazioni che comporta il ricorso ai differenti artefatti che articolano la realtà e il mondo sociale. È il principio della prevalenza del mezzo sul fine, sul quale si fonda l'ermeneutica materiale weiliana.

La materialità richiamata da Weil è in primo luogo il sistema degli oggetti che popola il mondo abitato dal vivente. Di conseguenza, la prima tappa di ogni sistema di oppressione, secondo Weil, non può che essere un determinato utilizzo della materia. Il capitalismo, e in particolare il processo di automatizzazione meccanica del lavoro, ha avuto una prima tappa "vecchia come l'umanità", che è consistita nell'aver affidato "a oggetti collocati in luoghi opportuni tutti gli sforzi di resistenza finalizzati a impedire certi movimenti da parte di certe cose" (Weil 2015, p. 30). Agli occhi di Weil il fine di questo sfruttamento delle potenzialità degli oggetti materiali è, ovviamente, ideologico. La materia inerte non può essere colpevolizzata, in quanto non può essere considerata un agente morale. La responsabilità morale e politica, poiché va di pari passo con l'intenzionalità, ossia con l'agire in base a un determinato scopo, è un monopolio dell'essere umano, e trasferirla agli oggetti non è nient'altro che un trucco di prestigio facilmente smascherabile.

Questa irresponsabilità della materia inerte, che con la sua inerzia oppone una resistenza e un attrito all'agire umano, diventa dunque un potente strumento di oppressione quando viene utilizzata per mascherare la responsabilità umana: "si tratta sempre di affidare alla materia quel che sembrava essere il ruolo dello sforzo umano, ma invece di utilizzare l'energia fornita da certi fenomeni naturali, si utilizzano la resistenza, la solidità, la durezza posseduta da certi materiali" (Weil 2015 , p. 29). Questo trasferimento di responsabilità è tuttavia ideologico, giacché tende a mascherare il ruolo sempre attivo dell'agente umano: "mai nessuna tecnica - prosegue Weil - dispenserà gli uomini dal rinnovare e adattare continuamente, col sudore della fronte, gli attrezzi di cui si servono" (Weil 2015, p. 31).

Vorrei insistere su questo rapporto di delega della responsabilità alla materia individuato da Weil facendo riferimento alle riflessioni sulla tecnica di Günter Anders. Nel primo volume de L'uomo è antiquato, Anders descrive il rapporto tra la specie umana e il mondo degli oggetti tecnici nei termini di una vergogna prometeica (Prometheische Scham). Essa è il sentimento che colpisce l'individuo di fronte ai prodotti della tecnica. Il "dislivello prometeico" che questo sentimento di vergogna registra è dovuto al fatto che l'uomo si riconosce ontologicamente inferiore rispetto agli oggetti tecnici e, per questo motivo, completamente in balia del loro funzionamento. La differenza fra gli umani e gli oggetti è radicale e apparentemente incolmabile. Mentre ogni essere umano è unico, irripetibile e necessariamente mortale, gli oggetti tecnici nell'era della loro riproducibilità sono ripetibili e, dunque, potenzialmente immortali. Questa onta viene lavata attraverso 
il tentativo dell'essere umano di divenire-oggetto: questo tentativo si realizza da un lato attraverso le tecniche di Human Engineering quali il make-up e, potremmo aggiungere noi, la chirurgia estetica o le tecniche di body empowerment, dall'altro lato mediante un'ossessiva riproduzione di immagini, ciò che Anders definisce iconomia (Ikonomanie). L'immagine infatti, proprio come gli oggetti che provocano la vergogna prometeica, è potenzialmente riproducibile all'infinito. Le strategie dell'umano per eliminare il sentimento di vergogna prometeica sono dunque trasformarsi in oggetto o trasformarsi in immagine. In entrambi i casi, l'umano denuncia il suo essere antiquato.

Per essere più precisi, ciò di cui l'uomo si vergogna è il suo natum esse, il suo essere legato irreparabilmente, in quanto corpo che deve necessariamente nascere per essere-nel-mondo, all'elemento biologico. Proprio l'irresponsabilità dell'essere nato è ciò che produce il sentimento di vergogna, così come il gobbo - l'esempio è di Anders - si vergogna della sua gobba proprio perché non ne può essere responsabile (Anders 2007, p. 96). A questa vergogna si va ad aggiungere, in un processo cumulativo, la vergogna di non potersi realmente trasformare in oggetto, ossia di essere proprio in quanto uomo ontologicamente mancante rispetto agli oggetti tecnici prodotti dallo stesso genere umano.

In questo contesto Anders inserisce un'interessante analisi del problema della responsabilità all'epoca della vergogna prometeica, usando come esempio la storia del generale MacArthur. Durante la guerra in Corea, venne deciso di sottrarre a MacArthur il monopolio della decisione se entrare in guerra o meno, delegandolo ai processi algoritmici di una macchina. Il cervello elettronico diede fortunatamente risposta negativa, ma questo è un aspetto secondario. Il punto filosofico centrale di questa vicenda storica, che implica una riflessione sul trasferimento di responsabilità dall'umano all'universo artefattuale, è così descritto da Anders:

ormai non è più un segreto per nessuno che al principio del conflitto coreano il generale McArthur propose delle misure la cui esecuzione avrebbe anche potuto scatenare una terza guerra mondiale. Ed è del pari noto a tutti che la decisione se si dovesse rischiare o meno tale conseguenza gli fu tolta dalle mani. Coloro però che gli tolsero questa responsabilità non lo fecero per assumere essi stessi la decisione o per addossarla a uomini più qualificati in campo politico, economico o morale; ma (dato che «l'ultima parola» doveva essere oggettiva e che «oggettive» sono considerate oggi soltanto le enunciazioni degli oggetti), per inoltrarla a una macchina - insomma: si trasmise la responsabilità decisiva a un Electric Brain (Anders 2007, p. 89).

Trasferendo per la prima volta risolutamente la decisione dall'umano alla macchina in virtù delle infinitamente maggiori capacità di giudizio oggettivo di quest'ultima, il genere umano ha inaugurato una nuova epoca che coincide, forse, con l'inizio della propria fine. Anders ammette che si sarebbe portati a dire che tale trasferimento è valso il mantenimento dell'essere umano stesso, giacché la macchina ha optato per una decisione negativa che ha permesso di preservare il genere umano. Allo stesso tempo però l'uomo ha consegnato nelle mani di ciò che è radicalmente altro da lui la propria stessa esistenza, scendendo così nella piramide dell'essere. Così Anders:

chi trasferisce la responsabilità da un uomo a una macchina trasferisce con ciò anche la sua propria responsabilità; $[\ldots]$ in quell'occasione, per la prima volta, l'umanità effettuò 
in modo emblematico la propria degradazione; per la prima volta dichiarò a se stessa pubblicamente: «dato che contiamo meno bene della nostra macchina, non si può far conto su di noi; dunque noi non 'contiamo'»; per la prima volta, non sentì l'umiliazione di umiliarsi pubblicamente (Anders 2007, p. 90).

Tuttavia, questo trasferimento di responsabilità sembra essere irrealizzabile una volta per tutte in maniera definitiva. Come abbiamo visto, Weil aveva già sottolineato questo punto: il trasferimento di responsabilità agli oggetti serve unicamente a mascherare l'inestirpabile responsabilità umana. Questo perché la decisione stessa di trasferire la propria responsabilità altrove, negli oggetti o nella serie indecifrabile di algoritmi di un processore, è per l'appunto una decisione umana, ed è essa stessa un esercizio della responsabilità e della libertà umane. Insieme al fardello del natum esse l'uomo porta con sé anche l'impossibilità a delegare una volta per tutte alla materia le sue istanze.

Questo trasferimento non è solo impossibile, ma è anche altamente problematico. La macchina a cui si sono rivolti i detrattori di MacArthur infatti, proprio in virtù della sua logica oggettiva, non è in grado di computare questioni morali: "intrinseca alla macchina è la sua idée fixe, cioè: la limitazione artificiale delle sue determinanti, il fatto che il punto di vista da cui rielabora il materiale viene regolato in anticipo e quindi resta fisso una volta per tutte" (Anders 2007, p. 90). Per poter trasferire in questo sistema fisso di elaborazione dati la responsabilità umana "si rinunciò a priori alle domande morali" (Anders 2007, p. 91), ossia alla caratteristica propria del genere umano: mettere in questione non l'efficacia strumentale del perseguimento di un fine, bensì il fine stesso. La materia, qualunque sia la forma tecnica nella quale è declinata, è irresponsabile perché la responsabilità contempla una riflessione non solo sulla relazione fra il mezzo e il fine, ma anche sulla loro natura. L'unica razionalità della macchina è la weberiana Zweckrationalität: razionalità rispetto allo scopo, adeguamento dei mezzi ai fini ma nessuna riflessione né sui primi né sui secondi. Una macchina sarà in grado di rispondere alla domanda sull'efficacia di determinati metodi di distruzione, ma non sul concetto in sé di distruzione. Su ciò, solo l'umano è capace di interrogarsi.

Tenendo insieme le posizioni di Weil e Anders, due risultano dunque essere i motivi per cui il trasferimento di responsabilità dall'umano all'artefattuale non è né perseguibile in via definitiva né auspicabile: 1) esso presuppone sempre, in un certo punto del processo, una decisione umana, e perciò l'umano non può essere una volta per tutte espulso dal sistema-mondo: esso coesiste con gli oggetti che lo circondano e che permettono l'esistenza; 2) comporta la rinuncia a qualsiasi questione morale.

Anders, in scritti posteriori più militanti, ritornerà sulla questione della responsabilità. Interrogandosi su quale dovesse essere la soluzione politica da attuare nei confronti di coloro che, detentori di armamenti nucleari, minacciavano di distruggere l'umanità intera, Anders insisteva sulla prerogativa umana di tale decisione. L'obiettivo polemico non deve essere l'oggetto in sé, bensì chi lo costruisce e chi ne conserva il monopolio. Vi è insomma una priorità politica dell'umano sull'artefattuale, un'attualità che nessuna vergogna prometeica può rovesciare. Nell'epoca della riproducibilità tecnica, distruggere gli oggetti potenzialmente letali per la specie umana non è solo particolarmente complicato a causa della loro struttura, ma anche inutile (perché sono riproducibili) e insensato (perché la materia è irresponsabile): "è come se una donna, aggredita da suo marito con un coltello, lo minacciasse di rompergli il coltello" (Anders 2008, p. 30). 
Le riflessioni di Weil e di Anders convergono su alcuni punti. Determinati oggetti (per Anders l'intero sistema degli oggetti) sono i primi elementi di un sistema di dominio, giacché organizzano lo spazio in modo da permettere determinate azioni e negarne altre. Essi, per essere più precisi, articolano politicamente l'ambiente in virtù delle loro proprietà materiali. È appena il caso di sottolineare come oggigiorno strumenti come muri e fili spinati organizzino l'attraversamento delle frontiere, ossia la mobilità umana, in virtù primariamente della loro struttura fisica. Questi ragionamenti non sono dunque astratti, ma immediatamente applicabili alla nostra realtà politica.

Le posizioni di Weil e Anders non sono tuttavia perfettamente sovrapponibili. Mentre secondo Weil l'organizzazione materiale è sempre un'opera umana che produce le condizioni di possibilità per il dominio di altri esseri umani, Anders ha una visione più manichea, perlomeno in L'uomo è antiquato, che isola la specie umana e la contrappone al sistema degli oggetti. Ad ogni modo, piuttosto che sulle divergenze teoriche, vorrei insistere sul fatto che la loro riflessione mostra come la responsabilità sia un tema fondamentale nell'organizzazione materiale del mondo, nella produzione di oggetti. Se gli artefatti hanno proprietà implicite nella loro materialità, questo comporta un'assunzione di responsabilità da parte di coloro che contribuiscono alla loro produzione. D'altronde, come entrambi gli autori rilevano, la responsabilità, in quanto connessa all'agency morale dell'umano, non è mai completamente trasferibile.

\section{La politicità dell'artefatto}

Il metodo materialista procede a un'analisi dei rapporti di dominanza attraverso la materia privo di una esplicita teoria ontologica su cui meglio fondare tale inversione del rapporto hegeliano fra lo spirito e la sua oggettivazione. A tal fine, vorrei introdurre come complemento al ragionamento finora svolto la riflessione ontologica sugli artefatti. In seguito, verificherò in che modo anch'essa presenti un possibile risvolto politico.

Come abbiamo visto in precedenza, Aristotele aveva mostrato nella Fisica la separazione fra oggetti naturali e artefatti ${ }^{8}$. La totalità dei possibili oggetti che popolano il mondo non si riduce tuttavia a questo insieme, e ciò provoca dei problemi teorici. Rispetto alla distinzione con gli oggetti naturali sancita dal testo aristotelico, più problematica appare infatti la relazione tra artefatti e oggetti sociali (matrimoni, promesse, debiti, denaro, giuramenti). Entrambi costituiscono il mondo sociale nel quale l'essere umano si muove; entrambi derivano la loro esistenza dall'azione umana. Eppure, siamo portati a pensare che una sedia sia più simile a un tronco piuttosto che a un matrimonio. Dobbiamo dunque evidenziare in base a quali criteri procediamo a una classificazione di questi differenti tipi di oggetti.

\footnotetext{
Tale distinzione è stata problematizzata, fra gli altri, da Hodder. Hodder non nega la separazione ontologica fra persone e cose, ma invita a riflettere sulla loro ineliminabile interazione reciproca. Gli uomini dipendono dalle cose così come le cose dipendono dagli uomini, in modo tale da risultare non pensabile un mondo di cose privo di essere umani e un mondo di umani privo di cose. La distinzione ontologica dunque può essere mantenuta, ma con la consapevolezza che un approccio pragmatista in senso lato non può considerare mai le due dimensioni come realmente autonome (Hodder 2012, pp. 88-112).
} 
Una prima distinzione tra artefatti e oggetti sociali potrebbe far capo alla considerazione per cui la produzione di oggetti sociali è un'azione propriamente umana, mentre la produzione di artefatti accomuna il vivente tout court. Appare difficile contestare che la diga costruita da un branco di castori, così come un termitaio o un alveare, siano a tutti gli effetti artefatti (Gould 2007). Tuttavia, anche le danze di accoppiamento che numerose specie animali compiono, e che hanno messo in crisi i numi tutelari dell'etologia (Lorenz 1965; Eibl-Eibesfeldt 1967), potrebbero essere interpretate come oggetti sociali. Questa distinzione interspecifica risulta dunque quantomeno problematica. Vi è tuttavia un altro argomento citato con frequenza nella letteratura sul tema che mi sembra utile per il tema trattato.

Nonostante le diversità delle varie posizioni, la ricerca filosofica sugli artefatti condivide generalmente il principio per cui l'artefatto è dotato di una autonomia ontologica rispetto agli oggetti sociali e socializzati ${ }^{9}$. Tale autonomia si fonda sulla prevalenza della costituzione materiale. L'autonomia ontologica che il metodo materialista sottintende senza mai esplicitare deriva dunque dalle proprietà materiali che costituiscono l'artefatto e che sono determinanti per la sua capacità di organizzare lo spazio vitale degli esseri umani. Diego Marconi sintetizza così la specificità degli artefatti rispetto ad altri tipi di oggetti: "i poteri causali degli oggetti sociali dipendono per la loro persistenza dalla persistenza (implicita o esplicita) delle intenzioni dei loro creatori, mentre i poteri causali degli artefatti, come quelli degli oggetti naturali, non ne dipendono" (Marconi 2016, p. 217). Mentre dunque un oggetto sociale soddisfa determinate funzioni in forza per alcuni autori di un consenso collettivo, per altri del valore performativo degli atti iscritti, ossia dei documenti che lo producono, le funzioni di un artefatto e le sue proprietà causali procedono dalla sua base materiale e dalle sue modalità di assemblaggio (Nail 2017) ${ }^{10}$.

A quale genere di funzioni e proprietà causali solitamente fanno riferimento $\mathrm{i}$ teorici dell'artefattualità? Funzioni e proprietà molto semplici e immediatamente individuabili, che apparentemente nulla hanno a che spartire con l'afflato politico che anima il metodo materialista, volto a determinare le leggi di funzionamento e le forme di dominio di strutture sociali altamente complesse. La caffettiera, un esempio classico di questa letteratura, è progettata in modo da avere la funzione di fare il caffè, e manterrà tale funzione nonostante qualunque credenza e rappresentazione sociale su di essa - a differenza, ad esempio, di un confine politico, il quale cessa di esistere nel momento stesso in cui viene a mancare un consenso collettivo sulla sua effettiva esistenza. Probabilmente a causa di evidenti difficoltà analitiche nel

9 Su questo punto, e in generale sulla distinzione tra oggetti sociali e oggetti naturali, si veda Ferraris 2009, pp. 50-56.

10 È opportuno a questo punto introdurre una distinzione metodologica tra funzione attuale e funzione potenziale. Solitamente le teorie degli artefatti fanno riferimento alle funzioni potenziali di un artefatto, ossia a quelle funzioni la cui attivazione è sempre regolata dalla mano umana. La matrice di tale distinzione è ovviamente aristotelica. Una caffettiera ha in potenza la funzione di fare il caffè, ma tale funzione per essere attivata necessita sempre dell'azione umana. Allo stesso tempo, benché più rari, esistono artefatti che non necessitano di essere attivati dalla mano umana dopo essere stati prodotti. La loro materializzazione implica il loro funzionamento. Un recinto di filo spinato, ad esempio, mantiene sempre attiva la funzione di separare lo spazio. La peculiarità di tali oggetti consiste nel fatto che la loro struttura materiale è sovraordinata rispetto alla loro funzione. In essi la funzione potenziale è sempre attualizzata, ossia potenza e atto coincidono senza resti. La letteratura su questo secondo tipo di artefatti, certamente rari ma pur sempre presenti nel nostro mondo sociale, è purtroppo estremamente carente, se non nulla. 
tracciare le funzioni implicite di un oggetto complesso ${ }^{11}$ - ad esempio: una telecamera a infrarossi -, i teorici degli artefatti sono sempre restii a porsi la questione su quali possano essere le implicazioni politiche di un artefatto, ossia su quali rapporti di potere e dominanza accompagnino necessariamente un artefatto. Tuttavia, dal punto di vista strettamente teorico che vorrei mettere a tema in questa sede, la gradazione di complessità fra due oggetti non inficia in alcun modo l'ipotesi per cui un artefatto organizza il Lebensraum del vivente sempre e comunque in virtù delle sue proprietà fisiche. Ciò equivale a dire che qualunque oggetto possiede una determinata capacità normativa. Vi è tutto un portato etico e politico di tale estensione della teoria degli artefatti, che ben si sposa con le analisi materialiste trattate in precedenza. Se infatti gli artefatti costruiti per mano umana svolgono determinate funzioni in base alla loro costituzione materiale, allora è precisamente compito della responsabilità umana, che mai può essere derogata alla materia inerte, progettare un sistema di oggetti tale da impedire o rendere disagevole il prodursi di determinati rapporti di dominio. La normatività degli oggetti non sottrae dunque responsabilità all'essere umano, ma viceversa la esalta.

Non sono molti gli autori ad aver indagato da una prospettiva filosofica le implicazioni del rapporto tra artefatti e potere. Fra essi, il filosofo della tecnologia Langdon Winner fu il primo, in un saggio del 1980, a tematizzare il rapporto che lega ontologicamente un artefatto e le sue implicazioni politiche. Winner sostiene che esistano determinati artefatti dotati di proprietà politiche in quanto artefatti. Essi istituiscono relazioni di potere non in virtù del ruolo che gli agenti sociali gli riconoscono, ma esclusivamente in virtù della loro costituzione materiale. "La bomba atomica - scrive Winner - è un artefatto intrinsecamente politico (inherently political artifact). Finché essa esiste nella sua forma attuale, le sue proprietà letali richiedono che essa venga controllata da una catena di comanda centralizzata e rigidamente gerarchica, chiusa a tutte quelle influenze che potrebbero rendere i suoi funzionamenti imprevedibili" (Winner 1980, p. 131). Evidentemente, la validità di un tale argomento suffragherebbe e renderebbe ragione di quel passaggio di Weil dove viene tematizzato il principio della prevalenza del mezzo sul fine e che compendia il metodo materialista weiliano. L'idea di Weil per cui la dialettica fra mezzi e fini è in realtà un movimento di explicatio, ossia che i fini sono l'esplicazione di determinate proprietà implicite nei mezzi, sembra risultare una mera petizione di principio se assunto semplicemente nella forma di un principio strategico e metodologico di analisi; la tesi di Winner, viceversa, contribuisce a fornirle una solida ossatura teorica.

Non sono certo mancate le critiche alla posizione di Winner, definita da Philip Brey "realismo radicale" (Brey 2005). La tesi dell'inherently political artifact è problematica perché, se portata alle sue estreme conseguenze, comporta una estromissione dell'azione umana dalla costruzione della realtà sociale. Per evitare che l'uomo sia misura di tutte le cose, il realismo winneriano finisce con il sostenere che le cose siano la misura dell'uomo. Va sottolineato che la posizione di Winner è in realtà maggiormente sfumata. Temendo di assegnare una normatività eccessiva all'oggettuale, sacrificando così il lato dell'azione umana e la relativa responsabilità morale, Winner ha affiancato alla sua tesi principale una posizione più moderata, volta a tenere insieme sia il ruolo performativo degli oggetti in sé, sia il ruolo degli umani nella definizione delle funzioni politiche degli artefatti. Sulla scia di una tradizione

11 Per un tentativo in questo senso si veda Chamayou 2013. 
di pensiero che tiene insieme Friedrich Engels, Peter Kropotkin, William Morris, Lewis Mumford e altri autori, Winner sostiene che determinati artefatti risultano compatibili con "determinate forme di relazioni politiche" (Winner 1980, p. 123) piuttosto che con altre. L'organizzazione del mondo mediante determinati artefatti può essere più o meno democratica, più o meno egualitaria, più o meno inclusiva: questo "più o meno" dipende dalla capacità normativa degli oggetti ma non espelle del tutto la componente umana. Questa posizione mi sembra un buon compromesso tra la radicalità del realismo e quella del costruttivismo: essa riconosce che gli oggetti hanno proprietà performative che derivano dalla loro costituzione fisica, ma allo stesso tempo sottolinea che la scelta di organizzare il mondo in modo da rendere possibili determinate relazioni politiche piuttosto che altre non può essere in alcun modo delegata: essa rimane appannaggio dell'essere umano.

Lo stesso argomento può essere esteso all'analisi dell'oggetto in sé. Determinati oggetti permettono un gioco delle rappresentazioni sociali più ampio rispetto ad altri. La bomba atomica ad esempio implica una gestione necessariamente autoritaria, poiché le sue proprietà causali comportano effetti distruttivi tali da non renderla una tecnologia adatta a un uso condiviso. Altri artefatti - muri, ponti, catene di montaggio, porte scorrevoli, telecamere di sorveglianza -, pur mantenendo determinate proprietà normative, sono aperti a rappresentazioni sociali e culturali, e confermano che gli esseri umani mantengono sempre la capacità di negoziare fra di loro le funzioni politiche da attribuire alle cose. Un muro può rappresentare una violenta separazione tra due popoli, ma può allo stesso tempo essere uno spazio di pittura aperto a tutti. Una telecamera di sorveglianza può ispirare una volontà di controllo totale e oppressiva sulle vite, ma può anche servire a monitorare luoghi oggettivamente insicuri in modo da permetterne una messa in sicurezza. L'artefatto rende possibile una determinata forma di rapporto con il mondo e con gli altri soggetti, ma questa forma è quasi sempre l'oggetto dell'attività ermeneutica dell'essere umano.

Uomini e oggetti coesistono, e non sembra né possibile né utile una svalutazione ontologica ed epistemologica dei secondi in favore dei primi. Vorrei ribadire che questa interpretazione dell'oggettuale non mira in alcun modo a una deresponsabilizzazione dell'azione umana. Anzi, a questo decentramento ontologico dell'essere umano si accompagna la sua affermazione come agente morale. Insisto su questo punto perché lo ritengo decisivo. La responsabilità dell'umano deve penetrare fin nel cuore del processo di produzione degli oggetti poiché è sempre l'umano a realizzarli o a deciderne la produzione $\mathrm{e}$, in questo modo, a favorire, per via della performatività della materia, determinate forme di esistenza rispetto ad altre. L'azione etica e politica fondamentale dell'essere umano è dunque l'organizzazione della materia, la produzione di un mondo di artefatti, di un sistema di oggetti destinato ad articolare l'esistenza dei viventi. Se dare vita a un mondo di oggetti destinato a essere abitato anche da altri non è un atto neutrale, allora un'ermeneutica materiale del mondo non può che considerare la dimensione oggettuale e la sua produzione come una delle poste in gioco più alte della prassi umana.

\section{Conclusioni: una filosofia politica e morale della materialità}

Il saggio ha presentato alcuni spunti di riflessione per elaborare una ermeneutica materiale del mondo attraverso una sintesi fra il metodo materialista e la teoria sugli 
artefatti. Dopo aver sostenuto la tesi della reciproca dipendenza di viventi e oggetti, ho mostrato come due linee di pensiero della tradizione filosofica occidentale, il materialismo e la teoria degli artefatti, possano sintetizzarsi in ragione dal campo del reale che condividono come oggetto privilegiato d'indagine: il mondo della datità materiale.

Le linee di ricerca aperte da tale sintesi convergono verso un ripensamento del ruolo dell' essere umano nel suo rapporto con il mondo. Tale rapporto è infatti già da sempre articolato da oggetti che lo mediano e lo rendono possibile. "La materialità degli oggetti - scrive Giovanni Leghissa - non è mero riflesso della cultura: la materialità non solo accompagna la capacità umana di produrre significati condivisi, ma in un certo senso la precede. [...] Si tratta insomma di non perdere mai di vista la concretezza del mondo materiale, la quale predelinea in modo inequivocabile $i$ percorsi percettivi e cognitivi dei soggetti agenti nel mondo" (Leghissa 2014, pp. 22-23).

Oltre a ciò, 1'essere umano nasce in un mondo di oggetti che sono stati prodotti da altri. Questo significa che dell'esperienza che attraverso gli oggetti l'essere umano può fare del mondo sono responsabili le generazioni passate. Nel riconoscimento delle proprietà performative degli artefatti vi è dunque anche il riconoscimento di una responsabilità transgenerazionale ${ }^{12}$. Gli oggetti, poiché sopravvivono ai loro costruttori, sono ciò che tiene insieme le generazioni, il filo che lega i padri ai figli. Vi è un'etica nell'uso e nell'organizzazione del mondo che abbraccia i secoli, coinvolge chi non è ancora nato e, in questo modo, stringe insieme il passato e il futuro.

Per concludere, vorrei ribadire una conseguenza teorica e politica cruciale che mi sembra emerga con chiarezza da quanto finora detto. Decentrare l'essere umano, scalzarlo dal più alto gradino ontologico, significa conferire piena responsabilità alle sue azioni. La contraddizione tra questi movimenti è solo apparente. Il mondo degli oggetti rappresenta una sorta di liquido amniotico dell'umano: in esso l'essere umano si sviluppa, grazie a esso si forma e diventa un individuo. La vita umana è un continuo corpo a corpo con il regno degli oggetti. Poiché tale regno è composto dagli artefatti che appartengono al nostro passato di specie, da quelli che costruiamo nel nostro presente, e da quelli che progettiamo per il futuro, il mondo degli oggetti è il regno della piena responsabilità del soggetto. L'essere umano nasce immerso in un mondo di oggetti che ne articolano e organizzano la vita; allo stesso tempo, l'uomo si adatta al mondo mediante la costruzione di oggetti. Questa interdipendenza porta con sé tutto un afflato etico e politico, che pertiene ai modi attraverso cui l'essere umano, mediante il rapporto che intrattiene con l'oggettuale, produce le condizioni di possibilità per una forma di vita con gli altri viventi che dipende necessariamente dai modi attraverso cui il suo Lebensraum viene modificato e plasmato.

Il rapporto dell'umano con il mondo delle "cose più prossime" (Heidegger 1986, p. 7) determina allo stesso tempo i rapporti interindividuali. Il sistema tecnologico degli artefatti, la sfera politica e quella morale, non sono dimensioni autonome, ma si intrecciano formando il mondo che abitiamo. Per questo motivo ritengo opportuno rifiutare sia una posizione radicalmente realista sia una posizione radicalmente costruttivista rispetto alla materialità degli oggetti. La posizione realista fondamentalmente ignora il valore delle rappresentazioni sociali degli artefatti e le 12 Un convincente tentativo di costruire una filosofia politica incentrata sul tema della transgenerazionalità è
Andina (2016). 
capacità ermeneutiche dell'umano (se un muro in quanto artefatto provoca dibattiti internazionali che vertono sulla sua legittimità o meno è perché viene interpretato come il simbolo di una determinata politica della separazione); dal canto suo, la posizione costruttivista ignora le costrizioni della realtà fisica e la sua normatività intrinseca.

Il compromesso tra questi due estremi permette un'analisi dei mezzi tecnologici, del sistema degli oggetti, e rende allo stesso tempo possibile il giudizio critico. Gli artefatti agiscono perché, come abbiamo visto, non sono bruta materia su cui l'essere umano riversa la sua volontà, autonomia, intenzionalità etc. Questo riconoscimento della normatività della materia comporta un'affermazione dell'umano come agente morale. L'uomo è responsabili delle sue azioni, e tra queste azioni rientra l'organizzazione di un sistema di oggetti tecnici che organizza in forme specifiche il mondo. Se ho provato a tracciare quantomeno le linee guida di una filosofia politica della materia è perché la politica non si limita all'espletamento di procedure elettorali e alla partecipazione a processi democratici, ma comprende tutto l'insieme di pratiche attraverso cui l'umano costruisce e determina una certa composizione materiale del mondo. Tale filosofia politica è allo stesso tempo una filosofia morale, perché riconoscere la normatività intrinseca degli artefatti non significa assegnare loro una forma di agency morale. La capacità morale, la valutazione dei contenuti e del senso di una determinata azione, rimane una prerogativa dell'essere umano; se gli oggetti hanno una normatività intrinseca, assegnare loro altresì il ruolo di agenti morali implicherebbe delegare anche la responsabilità. Abbiamo visto che tale delega è politicamente pericolosa, eticamente irresponsabile, logicamente impossibile.

Riconoscere che l'esistenza umana si relaziona necessariamente con un sistema di oggetti implica da un lato un decentramento ontologico dell'umano - perché la sua vita dipende dal sistema di oggetti nel quale è sin dalla sua nascita immerso -, e allo stesso tempo la riaffermazione del suo essere un agente morale, perché egli rimane l'unico soggetto a cui può essere imputato un uso e un'organizzazione responsabile o irresponsabile del mondo. La politicità della materia non è dunque in contraddizione con il ruolo specifico dell'essere umano nella costruzione del mondo. Tale ruolo, come abbiamo rilevato, è allo stesso tempo politico e morale, e non potrà mai prescindere dal riconoscimento della normatività degli oggetti con $i$ quali l'essere umano coesiste.

\section{Riferimenti bibliografici}

Anders, W. (2007): L'uomo è antiquato. Vol. 1: Considerazioni sull'anima nell'epoca della seconda rivoluzione industriale, tr. it. L. Dallapiccola, Torino, Bollati Boringhieri.

Anders, W. (2008): Il mondo dopo l'uomo: tecnica e violenza, tr. it. L. Pizzighella, Milano, Mimesis.

Andina, T. (2016): Ontologia sociale. Transgenerazionalità, potere, giustizia, Roma, Carocci.

Balibar, É. (1993): La philosophie de Marx, Paris, La Découverte.

Benjamin, W. (2012a): "L'arcobaleno. Dialogo sulla fantasia", in: Id., Aura e choc. Saggi sulla teoria dei media, a c. di A. Pinotti e A. Somaini, Torino, Einaudi.

Benjamin, W. (2012b): "L'opera d'arte nell'epoca della sua riproducibilità tecnica", in: Id., Aura e choc. Saggi sulla teoria dei media, a c. di A. Pinotti e A. Somaini, Torino, Einaudi.

Bodei, R. (2009): La vita delle cose, Roma-Bari, Laterza. 
Brey, P. (2005): “Artifacts as Social Agents", in H. Harbers (ed.), Inside the Politics of Technology. Agency and Normativity in the Co-Production of Technology and Society, Amsterdam, Amsterdam University Press.

Chamayou, G. (2013): Théorie du drone, Paris, La Fabrique.

Coccia, E. (2011): La vita sensibile, Bologna, Il Mulino.

Eibl-Eibesfeldt, I. (1967): Grundriß der vergleichenden Verhaltensforschung, München, Piper.

Ferraris, M. (2008): Storia dell'ontologia, Milano, Bompiani.

Ferraris, M. (2009): Documentalità. Perché è necessario lasciar tracce, Roma-Bari, Laterza.

Ferraris, M. (2012): "Esistere è resistere", in M. De Caro e M. Ferraris (a c. di), Bentornata realtà, Torino, Einaudi.

Foucault, M. (2010): Le parole e le cose. Un'archeologia delle scienze umane, tr. it. E.A. Panaitescu, Milano, Rizzoli.

Gould, J.L. (2007): “Animal Artifacts”, in E. Margolis e S. Laurence (eds.), Creations of the Mind. Theories of Artifacts and Their Representation, Oxford, Oxford University Press.

Heidegger, M. (1968): “L'origine dell'opera d'arte”, in: Id., Sentieri interrotti, tr. it. P. Chiodi, Firenze, La Nuova Italia.

Hodder, I. (2012): Entangled. An Archaeology of the Relationship between Humans and Things, Chichester, Wiley-Blackwell.

Latour, B. (2005): Reassembling the Social: an Introduction to Actor-Network-Theory, Oxford, Oxford University Press.

Leghissa, G. (2014): “Ospiti di un mondo di cose. Per un rapporto postumano con la materialità", Aut-Aut, 361, pp. 10-33.

Lorenz, K. (1965): Evolution and Modification of Behavior, Chicagno, University of Chicago Press.

MacKenzie, D. (1984): "Marx and the Machine", Technology and Culture, 25 (III), pp. 473 502, DOI: $10.2307 / 3104202$.

Marconi, D. (2016): “Artefatti e oggetti sociali”, in T. Andina e C. Barbero (a c. di), Ermeneutica, estetica, ontologia. A partire da Maurizio Ferraris, Bologna, Il Mulino.

Marx, K. (1970): Lineamenti fondamentali della critica dell'economia politica (II vol.), tr. it. E. Grillo, Firenze, La Nuova Italia.

McLuhan, M. (1990): Gli strumenti del comunicare, tr. it. E. Capriolo, Milano, il Saggiatore.

Nail, T. (2017): “What is an Assemblage?”, SubStance, 46 (I), pp. 21-37.

Rancière, J. (2000): Le partage du sensible, Paris, La Fabrique.

Salis, R. (2011): “Aristotele, Categorie, 14. Il movimento e le sue specie”, in M. Bonelli e F. Guadalupe Masi (a c. di), Studi sulle Categorie di Aristotele, Amsterdam, Adolf M. Hakkert.

Uexküll, J. (1934): Streifzüge durch die Umwelten von Tieren und Menschen: Ein Bilderbuch unsichtbaren Welten, Hamburg, Rowohlt.

Weil, S. (2005): "Riflessioni sulla guerra", in: Id., Sulla guerra. Scritti 1933-1943, tr. it. D. Zazzi, Milano, il Saggiatore.

Weil, S. (2015): Riflessioni sulle cause della libertà e dell'oppressione sociale, tr. it. G. Gaeta, Milano, Adelphi.

Winner, L. (1980): “Do Artifacts Have Politics?”, Daedalus, 109 (I), pp. 121-136. 